\title{
Revistas brasileñas de Educación en SCOPUS: un análisis bibliométrico
}

\author{
María José Madrid Martína \\ Noelia Jiménez-Fanjul ${ }^{\mathrm{b}}$ \\ Carmen León-Manterob \\ Alexander Maz-Machado ${ }^{\mathrm{b}}$ \\ aUniversidad Pontificia de Salamanca - UPSA, España \\ bUniversidad de Córdoba - UCO, España
}

ORIGINAL

\begin{abstract}
Resumen
Objetivo. El auge de la producción científica en los últimos años ha motivado la realización de diversos estudios bibliométricos. En esta línea, este trabajo analiza las revistas brasileñas presentes en el índice de impacto de la base de datos SCOPUS, el SClmago Journal Rank, en la categoría de Educación entre los años 2000 y 2015.

Método. Para llevar a cabo este estudio, se descargaron de la base de datos SCOPUS los datos relativos a los documentos publicados en estas revistas en dicho período y, a partir del recuento de frecuencias, se han identificado los patrones de producción de documentos por revista y por año, las instituciones más productivas, los países más productivos y se ha determinado el grado de colaboración en la autoría, así como, el patrón de citación recibida.

Resultados. Los resultados muestran un aumento significativo de la producción de estas revistas, aunque de carácter muy local en esta comunidad científica y con un pequeño número de citas por documento.
\end{abstract}

Palabras Clave

Bibliometría; Brasil; Educación; Producción científica; Revistas; SCOPUS

\section{Brazilian Educational journals in SCOPUS: a bibliometric analysis}

\begin{abstract}
Objective. The increase of scientific production during the last few years has aimed to carry out different bibliometric studies. In this line, this study analyses the Brazilian journals included in the impact index of the SCOPUS database, SCImago Journal Rank, in the category Education from 2000 to 2015.

Method. In order to do this study, all the data related to the documents published in these journals during this period were downloaded from the SCOPUS database and frequency counts were made, they allowed to identify the patterns of production of documents by journal and by year, the most productive institutions and countries and to determine the degree of collaboration in authorship and the pattern of citation received.

Results. The results show a significant increase in the production of these journals, but with a very local source in this scientific community and with a small number of citations per document.
\end{abstract}

\section{Keywords}

Bibliometrics; Brazil; Education; Journals; Scientific production; SCOPUS

\section{Introducción}

El continuo aumento de la producción científica hace que los estudios bibliométricos cobren cada día más importancia. Estos estudios facilitan conocer cómo es la naturaleza de esta producción mediante la identificación de patrones de publicación, de colaboración o de impacto en términos de citación. La bibliometría permite obtener resultados cuantitativos y comparables sobre la comunicación escrita, por su estrecha relación con las matemáticas y la estadística (Montilla, 2012; Spinak, 1998). Esto permite que sea utilizada a la hora de diseñar tanto líneas de investigación como actuaciones en las políticas científicas de países, regiones o centros de investigación (Maz-Machado, Jiménez-Fanjul y Villarraga, 2016). 
Generalmente, los estudios sobre la difusión de la ciencia centran su atención en los países industrializados. Sin embargo, en los últimos años, debido a la incorporación a las bases de datos de la Web Of Science (WoS) y de SCOPUS de revistas de otras regiones, el análisis de dichas regiones periféricas se ha incrementado, dando mayor visibilidad y acceso a la literatura de dichas regiones y contribuyendo a la supresión del fenómeno conocido como ciencia perdida (Ayuso y Martínez, 2004; Gibss, 1995).

En particular, la producción científica latinoamericana se ha estudiado desde el punto de vista de la bibliometría con diferentes perspectivas. Por ejemplo, De Moya-Anegón y Herrero-Solana (1999) realizaron un estudio para determinar cuáles pueden ser los indicadores de esta región. La productividad en la economía y los campos de la administración en la región también fueron objeto de análisis (Koljatic y Silva, 2001), así como, la cooperación y el desarrollo de la ciencia mediante indicadores bibliométricos (De Moya-Anegón y Herrero-Solana, 1999; Fernández, Gómez y Sebastián, 1998).

Una línea de investigación en aumento es la que analiza un conjunto determinado de revistas de Latinoamérica, bien por países o por temáticas. Se han tratado de identificar cuáles pueden ser los patrones de citación y la visibilidad en las diversas bases de datos (Gómez, Sancho, Moreno y Fernández, 1999; Miguel, 2011; Miguel y Herrero-Solana, 2010); también se ha analizado la producción de educación en enfermería en Brasil en la base de datos LILACS detectándose que predominan los estudios cualitativos (Prado, Medina-Moya y Martínez-Riera, 2011). Así mismo, se ha investigado el impacto y la productividad latinoamericana sobre educación matemática (Torres-Alfonso, Peralta-González y Toscano-Menocal, 2014), entre otras cuestiones. Algunos estudios han determinado el índice h para determinadas revistas colombianas (Romero-Torres, Acosta-Moreno y TejadaGómez, 2013) o abogan por que dicho índice no es aplicable a las revistas latinoamericanas debido al sesgo de idioma en muchas de las bases de datos internacionales (Monge-Nágera, 2014). Otros estudios analizan el factor de impacto de las revistas de una región (Téllez-Zanteno, Morales-Buenrostro y Estañol, 2007), de un país especifico como Brasil (Coura y Willcox, 2003), o la colaboración en las revistas iberoamericanas de la categoría Information Science \& Library Science indexadas en el Journal Citation Reports (JCR) Social Science Edition (Maz-Machado, Jiménez-Fanjul y Madrid, 2015).

En ocasiones, el objeto de estudio es una sola revista latinoamericana (Krauskopf, 2012; Maz-Machado, Jiménez-Fanjul, Adamuz-Povedano y Bracho-López, 2015) o todas las de un país. Por ejemplo, se han estudiado las revistas de medicina publicadas en Argentina en los años 2007-2008 y su visibilidad internacional (Colombo, 2009) y Gálvez (2006) analizó y comparó las revistas biomédicas chilenas con respecto a todas las de Latinoamérica.

La base de datos SCOPUS tiene una gran cobertura de revistas publicadas en un idioma diferente al inglés, por lo que es adecuada para estudiar el comportamiento de la difusión del conocimiento científico latinoamericano a través de las revistas de esta zona. Aun así, en muchos campos, el número de revistas no anglosajonas no es amplio, por ejemplo, en la categoría Educación dentro de las ciencias sociales, hay 1066 revistas indexadas, pero solamente 32 son latinoamericanas. De estas 32 revistas, 18 son publicadas en Brasil, representando el $56,25 \%$ de todas la de Latinoamérica.

En definitiva, al tratarse de un pequeño número revistas y, considerando que las publicadas en Brasil suponen más de la mitad del total, estas son representativas de la producción latinoamericana en educación recogida en esta base de datos y de ahí su relevancia para el estudio. Por otra parte, no se conocen estudios específicos sobre este conjunto de revistas de Brasil. Lo que sí se ha investigado, en relación a este país, es la producción sobre determinadas áreas o temáticas, género o instituciones, pero, en todas las bases de WoS en su conjunto (Leta y Lewison, 2003; Ribas Jr, Moura, Soares, Gomes, y Bornstein, 2003; Tess, Furuie, Castro, Barreto, y Nobre, 2009). En cuanto a la producción educativa, se ha estudiado solamente a partir de los datos de los grupos de investigación registrados en el Conselho Nacional de Desenvolvimento Científico e Tecnológico de Brasil (Coutinho et al., 2012) sin ahondar en la producción educativa en las bases de datos de prestigio internacional, como lo son WoS y SCOPUS.

Se debe indicar que el mayor estudio internacional sobre revistas de educación lo realizaron Fairbairn et al. (2009), quienes estudiaron 1042 revistas de 15 bases de datos internacionales, agrupándolas en 26 disciplinas dentro del área educativa. Hallaron que el $46 \%$ de las revistas son estadounidenses y que ocho países publican el $95,6 \%$ de ellas, concluyendo que el factor de impacto es inadecuado para medir la calidad de las revistas de educación. 


\section{Objetivo}

El objetivo general de este estudio es realizar un análisis bibliométrico de las revistas de Brasil indexadas en SCOPUS en la categoría de Educación y con factor de impacto SJR del SCImago Journal Rank (http://www.scimagojr.com/) en el periodo de estudio. En particular se tienen los siguientes objetivos específicos:

1. Determinar el número de documentos publicados por las revistas brasileñas de educación con factor de impacto (SJR) indexadas en SCOPUS (categoría de Educación) en el período 2000 a 2015.

2. Identificar el tipo de documentos publicados en estas revistas.

3. Reconocer las instituciones y los países más productivos en el campo de la educación en estas revistas.

4. Establecer el grado de colaboración en este conjunto de revistas del campo de la educación.

\section{Materiales y método}

Es aceptado por la comunidad científica internacional, que las técnicas bibliométricas son herramientas adecuadas para realizar estudios de esta naturaleza, relacionados con los patrones de publicación en un conjunto de revistas de un campo o temática especifica. Para el estudio, se consultaron en la web de SCImago Journal \& Country Rank (SJR), en su edición de 2015, todas las revistas de la categoría Educación y se seleccionaron las correspondientes a Brasil (SCImago, 2007-2016). En total, se hallaron 18 revistas en esta base de datos. Posteriormente, se buscaron en la base de datos SCOPUS todos los documentos indexados de estas revistas publicados entre los años 2000 y 2015. La búsqueda se realizó durante el mes de julio de 2016. Se buscaron todos los registros a partir de los campos nombre de la publicación (Source title) y año de publicación (years [2000-2015]).

A partir de los resultados anteriores, se realizó una nueva revisión de las fuentes para excluir algunos congresos y libros que incluían nombres similares a las revistas buscadas. Finalmente se obtuvieron 6706 documentos publicados en un total de 18 revistas brasileñas de educación. Todos los datos se incorporaron a una base de datos ad hoc MS Access 2010, versión 14.0.7162.501, en la que se depuraron los datos y se extrajeron las diferentes tablas de frecuencia.

Fue necesario realizar procesos de normalización de nombres de las instituciones de los autores de los documentos para evitar fraccionamiento en la asignación de producción a nivel institucional, así como de los nombres de los países firmantes. Se siguió el método empleado en estudios anteriores similares a partir del campo de filiación institucional (Jiménez-Fanjul, 2016).

A partir del campo de filiación institucional ya normalizado se extrajo el país y la institución de cada autor, así como las instituciones y países firmantes de cada documento.

Se realizaron conteos de frecuencia de la producción atendiendo a varias variables, a saber: año de publicación, tipo de documento, instituciones, países y número de autores por documento. Se contabilizó el número de citas anual de cada documento y se relacionó con el número de autores del mismo.

Para obtener los patrones de colaboración, se contó el número de autores para cada documento y se halló el Grado de Colaboración (GC) para la coautoría, tanto total como anual. Se escogió el grado de colaboración establecido por Subramanyam (1983) y que se define para un conjunto $\mathrm{K}$ de documentos publicados en una revista como:

$$
\mathrm{GC}=1-\frac{f_{1}}{N}
$$

donde $\mathrm{f}_{1}$ = número de artículos con 1 autor en la colección $\mathrm{K}$.

$$
\mathrm{N}=\text { número total de artículos en dicha colección K. } N=\sum_{j} f_{j}
$$


Para el conteo de los autores de los documentos, se optó por el sistema de recuento completo, de tal forma que se tienen en cuenta todos los autores firmantes por igual asignándole a cada uno de ellos el documento de manera completa como indica Rousseau (1994).

\section{Resultados y discusión}

Las 18 revistas de Brasil publicaron un total de 6706 documentos de distintos tipos: artículos, editoriales, revisiones, notas, erratas, cartas, etc. Como se observa en la Tabla 1, ninguna de las revistas está en los dos primeros cuartiles del SJR 2015 (SClmago, 2007-2016), 10 están en Q3 y 8 en Q4. La revista Educação e Pesquisa fue la primera en ser indexada en SCOPUS en el año 2000. La más reciente incorporación en la base de datos es la revista Historia da Educação en el 2013. Además, 16 de las 18 revistas fueron indexadas a partir de 2006.

Así mismo, la que ha publicado la mayor cantidad de documentos es Interface: Comunicação, Saúde, Educação con 876 y la que menos es el Periódico Tchê Química, con solo 89. La media de documentos publicados por revista es 372,5 , sin embargo, existe gran variabilidad entre los datos debido a que el número de años en los que cada revista ha estado indexada y la periodicidad de estas son diferentes.

Por ello, se ha calculado el número de documentos por año de cada una de las revistas, resultando una media de 45,74 documentos al año, mediana de 44,2 documentos, con una desviación típica de 17,48. Por tanto el coeficiente de variación de estos datos es 0,38 , lo cual muestra cierta disparidad entre el número de documentos publicados por cada revista al año. Así tenemos algunas con una media anual alta como Revista Brasileira de Ensino de Física con 77,7 o Interface: Comunicação, Saúde, Educação con 73, mientras que otras tienen una media de publicaciones anual baja como Periódico Tchê Química con 17,8 o Meta: Avaliação con 19,1, se considera que esto se debe a que cada revista posee una periodicidad de publicación diferente. Las revistas Revista Brasileira de Ensino de Física y Interface: Comunicação, Saúde, Educação tienen una periodicidad trimestral, además la segunda tiene hasta 2 suplementos por año; mientras que las revistas Periódico Tchê Química y Meta: Avaliação tienen una periodicidad semestral y cuatrimestral respectivamente.

Tabla 1 - Número de documentos, índice de impacto y cuartil por revista.

\begin{tabular}{|l|c|c|c|c|c|c|}
\hline \multicolumn{1}{|c|}{ Revista } & $\begin{array}{c}\text { SJR } \\
2015\end{array}$ & $\begin{array}{c}\text { SJR } \\
\text { Cuartil }\end{array}$ & $\begin{array}{c}\text { Indexada } \\
\text { desde }\end{array}$ & $\begin{array}{c}\text { Documento } \\
\text { s }\end{array}$ & $\begin{array}{c}\% \text { de } \\
6706\end{array}$ & $\begin{array}{c}\text { Documentos por } \\
\text { año }\end{array}$ \\
\hline Interface: Comunicação, Saúde, Educação & 0,285 & Q3 & 2004 & 876 & 13,06 & 73,0 \\
\hline Cadernos de Pesquisa & 0,260 & Q3 & 2006 & 441 & 6,58 & 44,1 \\
\hline Educação e Sociedade & 0,244 & Q3 & 2006 & 621 & 9,26 & 62,1 \\
\hline Movimento & 0,220 & Q3 & 2010 & 402 & 5,99 & 67,0 \\
\hline Bolema - Mathematics Education Bulletin & 0,211 & Q3 & 2010 & 362 & 5,40 & 60,3 \\
\hline $\begin{array}{l}\text { RAE Revista de Administração de } \\
\text { Empresas }\end{array}$ & 0,207 & Q3 & 2007 & 359 & 5,35 & 39,9 \\
\hline Revista Brasileira de Educação Especial & 0,205 & Q3 & 2007 & 325 & 4,85 & 36,1 \\
\hline Revista Brasileira de Ensino de Física & 0,204 & Q3 & 2006 & 777 & 11,59 & 77,7 \\
\hline Revista Brasileira de Educação & 0,202 & Q3 & 2007 & 372 & 5,55 & 41,3 \\
\hline Ensaio & 0,190 & Q3 & 2007 & 349 & 5,20 & 38,8 \\
\hline Educação e Pesquisa & 0,171 & Q4 & 2000 & 581 & 8,66 & 48,4 \\
\hline
\end{tabular}


Tabla 1 - Número de documentos, índice de impacto y cuartil por revista (continuación).

\begin{tabular}{|l|c|c|c|c|c|c|}
\hline \multicolumn{1}{|c|}{ Revista } & $\begin{array}{c}\text { SJR } \\
2015\end{array}$ & $\begin{array}{c}\text { SJR } \\
\text { Cuartil }\end{array}$ & $\begin{array}{c}\text { Indexada } \\
\text { desde }\end{array}$ & $\begin{array}{c}\text { Documento } \\
\text { s }\end{array}$ & $\begin{array}{c}\% \text { de } \\
6706\end{array}$ & $\begin{array}{c}\text { Documentos por } \\
\text { año }\end{array}$ \\
\hline Educação e Pesquisa & 0,171 & Q4 & 2000 & 581 & 8,66 & 48,4 \\
\hline $\begin{array}{l}\text { Revista Brasileira de Orientação } \\
\text { Profissional }\end{array}$ & 0,168 & Q4 & 2011 & 127 & 1,89 & 25,4 \\
\hline Paideia & 0,165 & Q4 & 2011 & 224 & 3,34 & 44,8 \\
\hline Revista da Educação Física & 0,141 & Q4 & 2011 & 299 & 4,46 & 59,8 \\
\hline Cadernos CEDES & 0,111 & Q4 & 2006 & 235 & 3,50 & 23,5 \\
\hline Meta: Avaliação & 0,111 & Q4 & 2009 & 134 & 2,00 & 19,1 \\
\hline Periódico Tchê Química & 0,100 & Q4 & 2011 & 89 & 1,33 & 17,8 \\
\hline Historia da Educação & 0,100 & Q4 & 2013 & 133 & 1,98 & 44,3 \\
\hline
\end{tabular}

El número de documentos publicados ha aumentado notablemente, pasando de 17 documentos en el año 2000 a 987 en el 2015 (Tabla 2). Esto se debe a un aumento en la cobertura de las revistas regionales de Educación en la base de datos SCOPUS y por tanto al aumento del número de las mismas que se incluyen en el índice SJR. De hecho, el SJR pasa de indexar una única revista de este conjunto, la Educação e Pesquisa, entre los años 2000 y 2005 a concluir con las 18 revistas estudiadas. Hasta el año 2008 no se alcanza la decena de revistas en SJR para el conjunto estudiado. Además, en los años 2003 y 2005 no se halló ningún documento indexado.

De forma similar, lo anterior también se observa si se analiza la publicación con respecto a periodos determinados. Así, en el período que abarca desde el año 2000 al 2005, solo se publicaron el 0,82\% del número total de documentos. En ese mismo periodo se observa el pequeño número de revistas indexadas hasta esa fecha.

Entre 2006 y 2010 se publicaron un $32,19 \%$ del total de documentos de las revistas, y entre el 2010 y el 2015 , el $66,99 \%$ del total. Este incremento en la producción de documentos publicados en las revistas de educación de Brasil, es mayor que el hallado para las revistas españolas de educación (Maz-Machado, Jiménez-Fanjul y Adamuz-Povedano, 2014). La media de publicación es de 419,13 documentos por año.

Tabla 2 - Documentos por año entre 2000 y 2015.

\begin{tabular}{|c|c|c|c|}
\hline Año & № de documentos & \% de 6706 & Porcentaje acumulado \\
\hline 2000 & 17 & 0,25 & 0,25 \\
\hline 2001 & 18 & 0,27 & 0,52 \\
\hline 2002 & 19 & 0,28 & 0,80 \\
\hline 2003 & 0 & 0,00 & 0,80 \\
\hline 2004 & 1 & 0,01 & 0,82 \\
\hline 2005 & 0 & 0,00 & 0,82 \\
\hline 2006 & 213 & 3,18 & 3,99 \\
\hline 2007 & 389 & 5,80 & 9,79 \\
\hline 2008 & 418 & 6,23 & 16,03 \\
\hline 2009 & 533 & 7,95 & 23,98 \\
\hline 2010 & 606 & 9,04 & 33,01 \\
\hline
\end{tabular}


Tabla 2 - Documentos por año entre 2000 y 2015 (continuación).

\begin{tabular}{|c|c|c|c|}
\hline Año & № de documentos & \% de 6706 & Porcentaje acumulado \\
\hline 2011 & 824 & 12,29 & 45,30 \\
\hline 2012 & 814 & 12,14 & 57,44 \\
\hline 2013 & 913 & 13,61 & 71,05 \\
\hline 2014 & 954 & 14,23 & 85,28 \\
\hline 2015 & 987 & 14,72 & 100,00 \\
\hline Total & 6706 & 100,00 & \\
\hline
\end{tabular}

Con respecto al tipo de documento publicado, se observa que el documento predominante es el artículo científico, que supone el $88,88 \%$ de los documentos publicados. Así mismo, llama la atención que el número de documentos de tipo Editorial Material es mayor que el número de artículos de revisión, que se catalogan como Reviews en la base de datos SCOPUS (Tabla 3).

Tabla 3 - Tipo de documentos publicados.

\begin{tabular}{|l|c|c|}
\hline \multicolumn{1}{|c|}{ Tipo de documento } & № de documentos & \% \\
\hline Article & 5960 & 4,22 \\
\hline Editorial & 283 & 3,82 \\
\hline Review & 256 & 1,89 \\
\hline Note & 127 & 0,63 \\
\hline Conference Paper & 42 & 0,28 \\
\hline Erratum & 19 & 0,22 \\
\hline Letter & 15 & 0,06 \\
\hline Short Survey & 4 & 100,00 \\
\hline
\end{tabular}

Como dato anecdótico, algunas de las revistas de la muestra analizada han publicado comunicaciones de congresos (conference paper) de manera esporádica, de ahí el resultado que se refleja en la Tabla 3.

En estas revistas de la categoría Educación han publicado autores de 63 países diferentes, el 79,35\% de ellos son autores brasileños. El $84,43 \%$ de los documentos fueron escritos por autores de países latinoamericanos, mientras que España y Portugal han producido únicamente el 8,5\% de los documentos. Los países que se incluyen en la Tabla 4 han aportado el 98,08\% del total de documentos publicados en las revistas Brasileñas de Educación indexadas en SCOPUS y con factor de impacto SJR. Por tanto, casi la totalidad de los autores proceden de instituciones de países iberoamericanos, europeos y norteamericanos.

Tabla 4 - Número de documentos por país.

\begin{tabular}{|l|c|c|}
\hline \multicolumn{1}{|c|}{ País } & № documentos & \% de 6706 documentos \\
\hline Brasil & 5321 & 79,35 \\
\hline Portugal & 342 & 5,10 \\
\hline España & 228 & 3,40 \\
\hline USA & 131 & 1,95 \\
\hline Argentina & 99 & 1,48 \\
\hline Francia & 98 & 1,46 \\
\hline Chile & 96 & 1,43 \\
\hline
\end{tabular}


Tabla 4 - Número de documentos por país (continuación).

\begin{tabular}{|l|c|c|}
\hline \multicolumn{1}{|c|}{ País } & № documentos & \% de 6706 documentos \\
\hline Reino Unido & 92 & 1,37 \\
\hline Colombia & 51 & 0,76 \\
\hline México & 48 & 0,72 \\
\hline Italia & 36 & 0,54 \\
\hline Canadá & 35 & 0,52 \\
\hline
\end{tabular}

Las diez universidades más productivas publican el 45,57\% del total de documentos y todas ellas pertenecen a Brasil. La primera institución no brasileña en términos productivos, es la Universidade do Minho de Portugal situada en el puesto 22, con 72 documentos publicados, y, la primera universidad latinoamericana no brasileña, es la Universidad de Buenos Aires, con 35 documentos.

La Universidad Autónoma de Barcelona es la universidad española más productiva, con 23 documentos publicados y situada en el puesto 55 y, la primera universidad norteamericana, es la Stanford University, situada en el puesto 108.

Tabla 5 - Universidades más productivas en revistas educativas de Brasil en SCOPUS.

\begin{tabular}{|l|c|c|}
\hline \multicolumn{1}{|c|}{ Universidad } & № de documentos & $\%$ de 6706 \\
\hline Universidade de São Paulo & 729 & 10,87 \\
\hline Universidade Estadual de Campinas & 378 & 5,64 \\
\hline Universidade Federal do Rio Grande do Sul & 333 & 4,97 \\
\hline UNESP - Universidade Estadual Paulista & 325 & 4,85 \\
\hline Universidade Federal do Rio de Janeiro & 309 & 4,61 \\
\hline Universidade Federal de Santa Catarina & 249 & 3,71 \\
\hline Universidade Federal de Minas Gerais & 222 & 3,31 \\
\hline Universidade Federal de São Carlos & 186 & 2,77 \\
\hline Universidade do Estado do Rio de Janeiro & 169 & 2,52 \\
\hline Universidade Federal do Paraná & 156 & 2,33 \\
\hline
\end{tabular}

A partir del año 2008, el número de documentos en multiautoría empiezan a ser ligeramente mayores respecto a los de autoría única (Figura 1) hasta llegar a representar el 72,34\% de la producción del año 2015; coincidiendo también con el incremento de revistas de la muestra indexadas en SCOPUS. Esto puede ser consecuencia del auge en la implementación de programas de evaluación de la producción científica en las instituciones de educación superior, lo que hace que sea más provechosa la publicación en multiautoría, o también del aumento de las posibilidades para la colaboración que han surgido debido al desarrollo tecnológico. 


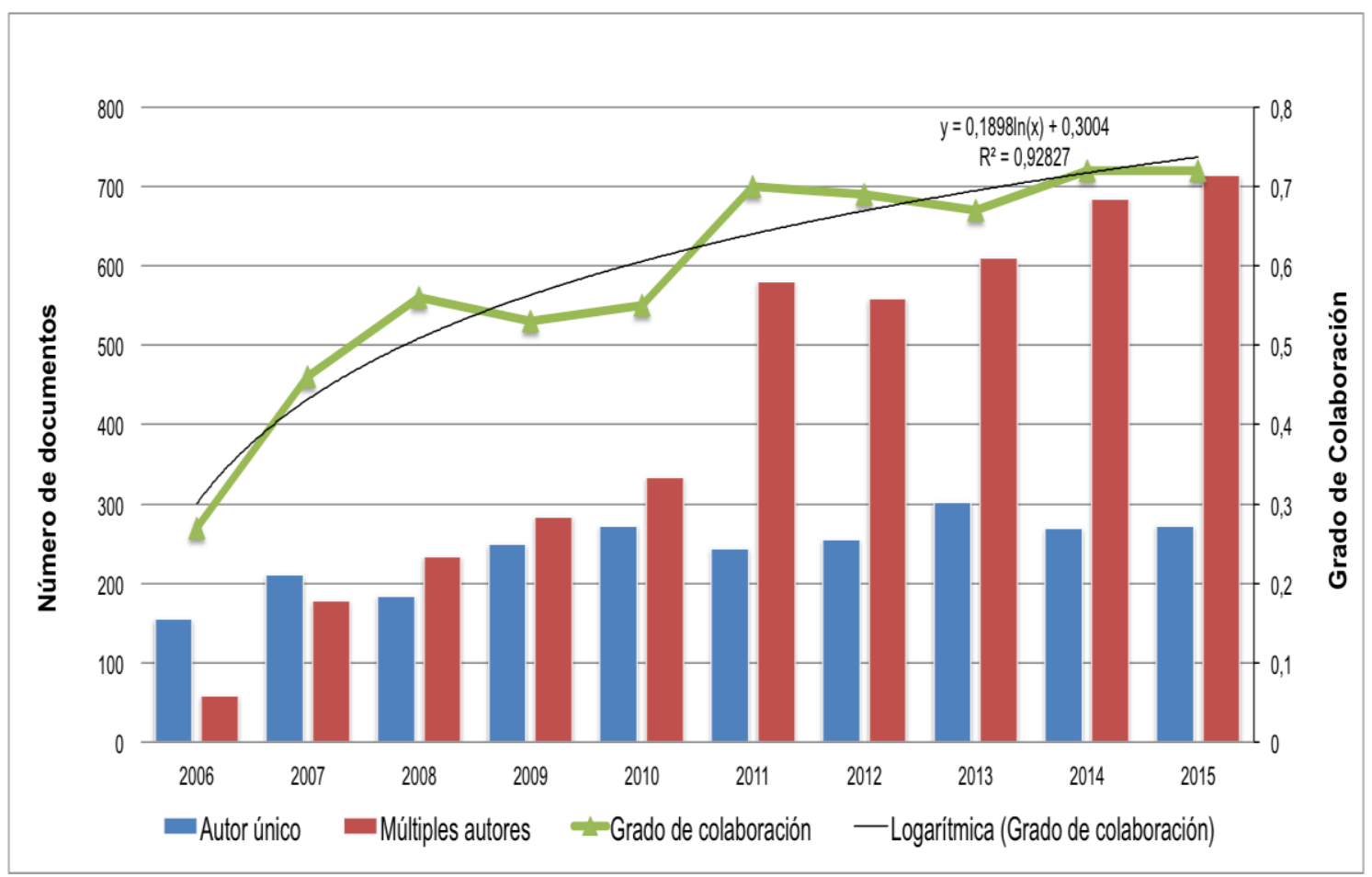

Figura 1 - Producción según el tipo de autoria: única o múltiple.

El 35,69\% de las publicaciones fueron firmadas por un solo autor, mientras que el 31,91\% las firman dos y el $17,82 \%$ tres. Así se tiene que el $49,73 \%$ del total de publicaciones son firmadas por 2 o 3 autores y tan solo 4 de los 6706 documentos considerados tienen 10 o más autores. Estos resultados contrastan con los hallados por Dess (2008) para la investigación educativa en Alemania donde el 58\% de las publicaciones en este campo tiene un solo autor.

Para determinar el Grado de Colaboración (GC) en la autoría, se excluyeron 113 documentos sin identificación de autor, así como los anteriores al 2006, porque en esos 5 años solo se publicaron 38 documentos con una sola firma. La tabla 6 presenta la evolución anual del GC en las 18 revistas analizadas, observándose un gran incremento del mismo. Además, si se comparan el año 2006 con el año 2008, en este pequeño periodo de tiempo el GC se duplicó, mientras que a partir de este momento el crecimiento se produce de forma más gradual produciéndose incluso períodos de decrecimiento como entre los años 2008 y 2009 o entre 2011 y 2013, o de estancamiento como en los años 2014-2015. De forma global, en el periodo 2006-2015, para las revistas de educación publicadas en Brasil incluidas en SCOPUS estudiadas, el valor del GC es 0,636.

Tabla 6 - Grado de Colaboración.

\begin{tabular}{|c|c|c|c|c|c|c|c|c|c|c|}
\hline Año & 2006 & 2007 & 2008 & 2009 & 2010 & 2011 & 2012 & 2013 & 2014 & 2015 \\
\hline $\begin{array}{c}\text { Grado } \\
\text { Colaboración }\end{array}$ & 0,27 & 0,46 & 0,56 & 0,53 & 0,55 & 0,7 & 0,69 & 0,67 & 0,72 & 0,72 \\
\hline
\end{tabular}

En total, los documentos de estas revistas han recibido 2386 citas en el periodo 2000-2015 y la tasa de citación promedio para un documento es de 2,81 citas. Esta tasa es similar a la resultante para la producción educativa en España entre 1998-2009 (Fernández-Cano, 2011), que alcanzó el 2,67, pero resulta inferior a los promedios de 3,7 citas por artículo hallado para la producción educativa en Suiza en WoS y de 7,9 en Google Scholar (Diem y Wolter, 2013). El 64,42\% de los documentos publicados en las revistas educativas de Brasil no han sido citados nunca (Tabla 7). El 17,68\% de los documentos han sido citados una única vez, mientras que solamente 5 documentos han recibido más de 20 citas. El 3,06\% de los documentos ha sido citado más de 5 veces. 
Si tenemos en cuenta la colaboración en la autoría de los documentos citados, aquellos firmados por un solo autor han recibido 1192 citas, es decir, que estos representan el 50,17\% del total de las citas. Resultado similar al encontrado para las revistas indexadas en SCOPUS y que publican artículos de educación matemática a nivel internacional, corroborando que la citación de un artículo disminuye según el número de autores que lo firman (Jiménez-Fanjul, 2016).

Tabla 7 - Citación a las revistas de educación de Brasil en SCOPUS.

\begin{tabular}{|c|c|c|}
\hline Citas & № & $\%$ \\
\hline 0 & 4320 & 64,42 \\
\hline 1 & 1192 & 17,78 \\
\hline 2 & 533 & 3,95 \\
\hline 3 & 251 & 1,97 \\
\hline 4 & 132 & 1,09 \\
\hline 5 & 73 & 0,81 \\
\hline 6 & 54 & 0,60 \\
\hline 7 & 40 & 0,39 \\
\hline 8 & 26 & 0,30 \\
\hline 9 & 20 & 0,21 \\
\hline 10 & 14 & 0,69 \\
\hline entre 11 y 20 & 46 & 0,07 \\
\hline mayor a 20 & 5 & 100,00 \\
\hline Total & 6706 & \\
\hline
\end{tabular}

\section{Conclusiones}

La producción científica sobre Educación en revistas de Brasil ha experimentado un significativo incremento durante los últimos años, especialmente a partir de 2006. Esto se debe en gran parte a la incorporación de nuevas revistas en SCOPUS, y por ende en el SJR, en los últimos años. Sin embargo, si se compara con los resultados presentados por Fairbairn et al. (2009), aún está muy lejos del número de revistas de educación que se publican en los países angloparlantes. Así mismo, se encuentran diferencias importantes en el número de documentos publicados por unas u otras revistas, destacando como mayores productoras la Revista Brasileira de Ensino de Física e Interface: Comunicação, Saúde, Educação. Como ya hemos apuntado, la periodicidad de las revistas y el período de indexación cubierto por SCOPUS tienen un impacto en el número de documentos que cada una de ellas aporta a la muestra analizada.

La citación en estas revistas sigue siendo poco relevante, solo el 17,82\% de los documentos ha recibido más de una cita, y esto afecta al impacto de estas, prueba de ello es que ninguna de ellas se encuentra en las categorías Q1 o Q2 del SCImago Journal \& Country Rank (SCImago, 2007-2016). Sería interesante analizar si esta tendencia se observa en otros estudios regionales y quizá valorar la repercusión que el alcance y política de la revista (regional o internacional) tiene sobre la citación de la misma. Es presumible que los estudios regionales y locales, mayoritariamente publicados por las revistas analizadas, tengan una audiencia limitada y por tanto su repercusión en el factor de impacto se vea sesgada; o quizás las revistas indexadas de educación en Brasil no sean el medio de difusión de resultados de investigación habitual en este campo, debiendo buscar la producción científica en revistas secundarias u otras fuentes.

Destaca que los documentos publicados son de un carácter altamente local, como lo señala que el 79,35\% sean firmados por autores de Brasil y la alta representatividad de sus universidades, lo que viene a corroborar que las revistas analizadas publican estudios locales o regionales de interés en los propios países que los realizan, lo que viene siendo habitual en las disciplinas propias de Educación. Además, el 92,23\% de los autores firmantes son iberoamericanos lo que muestra la poca relevancia que poseen estas revistas en el resto de países, quizás debido al idioma utilizado. 
Al inicio del periodo analizado las publicaciones eran producto de un autor que publicaba de manera aislada, pero esto ha cambiado de forma que actualmente los documentos en colaboración casi triplican a los individuales, tendencia habitual en distintos campos de investigación, quizá porque como señalan algunos investigadores los artículos en colaboración son una vía apropiada para aumentar el número de citaciones (Cañedo et al., 2016).

Estos resultados permitirán en un futuro comparar este conjunto de revistas de impacto brasileñas sobre educación con las de otros países o regiones sobre la misma temática y analizar si las tendencias aquí presentes son similares o si se trata de un caso particular.

\section{Referencias}

Ayuso, M. D., y Martínez, V. (2004). Propuesta de auditoria de recursos de información en Internet: SCIELO y la ciencia perdida. Investigación Bibliotecológica, 18(37), 85-109.

Cañedo, R., Nodarse, M., Cruz, J., Germán, I., Celorrio, I., y Guerrero, J. C. (2016). Papel de la colaboración científica en el impacto de la investigación en salud de Cuba en el contexto de América Latina. Revista Cubana de Información en Ciencias de la Salud, 27(1), 56-74.

Colombo, F. (2009). Visibilidad de las revistas argentinas de medicina en las bases de datos internacionales. Información, Cultura y Sociedad, 20, 41-62.

Coura, J., y Willcox, L. (2003). Impact factor, scientific production and quality of Brazilian medical journals. Memórias do Instituto Oswaldo Cruz, 98, 293-297.

Coutinho, R. X., Dávila, E. S., dos Santos, W. M., Rocha, J. B. T., Souza, D. O. G., Folmer, V., y Puntel, R. L. (2012). Brazilian scientific production in science education. Scientometrics, 92(3), 697-710.

De Moya-Anegón, F., y Herrero-Solana, V. (1999). Science in America Latina: a comparison of bibliometric and scientifictechnical indicators. Scientometrics, 46(2), 299-320.

Dees, W. (2008). Innovative Scientometric Methods for a Continuous Monitoring of Research Activities in Educational Science. Comunicación presentada en el Fourth International Conference on Webometrics, Informetrics and Scientometrics \& Ninth COLLNET Meeting, Berlin.

Diem, A., y Wolter, S. C. (2013). The use of bibliometrics to measure research performance in education sciences. Research in higher education, 54(1), 86-114.

Fairbairn, H., Holbrook, A., Bourke, S., Preston, G., Cantwell, R., y Scevak, J. (2009). A profile of education journals. Paper presented at the AARE 2008 International Educational Research Conference.

Fernández-Cano, A. (2011). Producción educativa española en el Social Sciences Citation Index (1998-2009). II. Revista Española de Pedagogía, 427-443.

Fernández, M. T., Gómez, I., y Sebastián, J. (1998). La cooperación científica de los países de América Latina a través de indicadores bibliométricos. Interciencia, 23(6), 328-337.

Gálvez, M. (2006). Publicaciones biomedicas: Realidad de Chile y latinoamerica. Revista Chilena de Radiología, 12(3), 113117.

Gibss, W. W. (1995). Lost science in the Third World. Scientific American, 273(2), 92-99. doi: 10.1038/scientificamerican089592

Gómez, I., Sancho, R., Moreno, L., y Fernández, M. T. (1999). Influence of Latin American journals coverage by international databases. Scientometrics, 46(3), 443.456.

Jiménez-Fanjul, N. (2016). Producción científica internacional en Educación Matemática. Estudio bibliométrico (1983-2012). Tesis doctoral, Universidad de Córdoba, Córdoba. Recuperado de http://hdl.handle.net/10396/13759

Koljatic, M., y Silva, M. (2001). The international publication productivity of Latin American countries in the economics and business administration fields. Scientometrics, 51(2), 381-394.

Krauskopf, E. (2012). Short term impact of the Chilean Journal of Agricultural Research. Chilean Journal of Agricultural Research, 72(1). doi: 10.4067/S0718-58392012000100026

Leta, J., y Lewison, G. (2003). The contribution of women in Brazilian science: A case study in astronomy, immunology and oceanography. Scientometrics, 57(3), 339-353. 
Maz-Machado, A., Jiménez-Fanjul, N., y Adamuz-Povedano, N. (2014). Spanish Journals of Education \& Educational Research in the JCR: A bibliometric analysis of the citations. Library Philosophy and Practice (e-journal), paper 1121.

Maz-Machado, A., Jiménez-Fanjul, N., Adamuz-Povedano, N., y Bracho-López, R. (2015). Análisis bibliométrico de la revista RELIME (1997-2011). Investigación Bibliotecológica, 29(86), 90-102.

Maz-Machado, A., Jiménez-Fanjul, N., y Madrid, M. J. (2015). Collaboration in the Iberoamerican Journals in the category Information Science \& Library Science in WOS. Library Philosophy and Practice (e-journal), paper 1270.

Maz-Machado, A., Jiménez-Fanjul, N., y Villarraga, M. (2016). La producción colombiana SciELO: un análisis bibliométrico. Revista Interamericana de Bibliotecología, 29(2), 15-26.

Miguel, S. (2011). Revistas y producción científica de America Latina y el Caribe: su visibilidad en SCiELO, RedALyC y SCOPUS. Revista Interamericana de bibliotecología, 34(2), 187-199.

Miguel, S., y Herrero-Solana, V. (2010). Visibilidad de las revistas latinoamericanas de bibliotecología y ciencia de la información a través de Google Scholar. Ciência da Informaçâo, 39(2), 54-67.

Monge-Nágera, J. (2014). La invalidez del factor de impacto como indicador de impacto de las revistas científicas latinoamericanas. Revista de Biología Tropical, 64(1), 407-412.

Montilla, A. (2012). Análisis bibliométrico sobre la producción científica archivística en la Red de Revistas Científicas de America Latina y el Caribe (Redalyc) durante el periodo 2001-2011. Biblios: Revista electrónica de bibliotecología, archivología y museología (48), 1-11. doi: 10.5195/biblios.2012.65

Prado, M. L., Medina-Moya, J. L., y Martínez-Riera, J. R. (2011). La producción del conocimiento en educación en enfermería en España y Brasil: una revisión integrativa. Texto \& contexto enferm, 20(3), 407-415.

Ribas Jr, R. C., Moura, M. L. S., Soares, I. D., Gomes, A. A. N., y Bornstein, M. H. (2003). Socioeconomic status in Brazilian psychological research: I. validity, measurement, and application. Estudos de Psicologia (Natal), 8(3), 375-383.

Romero-Torres, M., Acosta-Moreno, L. A., y Tejada-Gómez, M. A. (2013). Ranking de revistas científicas en Latinoamérica mediante el índice h: estudio de caso Colombia. Revista Española de Documentación Científica, 36(1), e003. doi:10.3989/redc.2013.1.876

Rousseau, R. (1994). The number of authors per article in library and information science can often be described by a simple probability distribution. Journal of Documentation, 50, 134-141.

SCImago. (2007-2016). 2015 SJR — SCImago Journal Rank Retrieved 20 de Julio de 2016, from http://www.scimagojr.com

Spinak, E. (1998). Indicadores cienciometricos. Ciencia de la Información., 27(2), 141-148.

Subramanyam, K. (1983). Bibliometric studies of research collaboration: A review. Journal of Information Science, 6, 33-38.

Téllez-Zanteno, J., Morales-Buenrostro, L., y Estañol, B. (2007). Análisis del factor de impacto de las revistas cientifica latinoamericanas. Rev Med Chile, 135(4), 480-487.

Tess, B. H., Furuie, S. S., Castro, R. C. F., Barreto, M. C. C., y Nobre, M. R. C. (2009). Assessing the scientific research productivity of a Brazilian healthcare institution: a case study at the Heart Institute of Sao Paulo, Brazil. Clinics, 64(6), 571-576.

Torres-Alfonso, A. M., Peralta-González, M. J., y Toscano-Menocal, A. (2014). Impacto y productividad de las publicaciones latinoamericanas sobre Matemática Educativa. Biblios: Revista electrónica de bibliotecología, archivología y museología (55), 13-26. doi: 10.5195/biblios.2014.161 


\section{Datos de los autores}

\section{María José Madrid Martín}

Graduada en Matemáticas por la Universidad de Salamanca. Doctora en Educación Matemática por la Universidad de Salamanca. Profesora de la Universidad Pontificia de Salamanca (España). mimadridma@upsa.es

\section{Noelia Jiménez-Fanjul}

Ingeniera en Automática y Electrónica Industrial por la Universidad de Córdoba. Doctora en Educación por la Universidad de Córdoba (programa de Ciencias Sociales y Jurídicas). Profesora del Departamento de Matemáticas de la Universidad de Córdoba (España).noelia.jimenez@uco.es

\section{Carmen León-Mantero}

Licenciada en Ciencias Matemáticas por la Universidad de Sevilla. Doctora por la Universidad de Córdoba en el programa de Ciencias Sociales y Jurídicas. Profesora del Departamento de Matemáticas de la Universidad de Córdoba (España).cmleon@uco.es

\section{Alexander Maz-Machado}

Licenciado en Matemáticas y Física por la Universidad de Tolima. Doctor en Ciencias Matemáticas por la Universidad de Ganada. Profesor del Departamento de Matemáticas de la Universidad de Córdoba (España). ma1mamaa@uco.es

Recibido - Received: 2016-07-29

Aceptado - Accepted: 2017-07-17

\section{$(\mathrm{cc})$ EY}

This work is licensed under a Creative Commons Attribution 4.0

United States License.

\section{UILIS D-Sorke}

This journal is published by the University Library System of the University of Pittsburgh as part of its D-Scribe Digital Publishing Program and is cosponsored by the University of Pittsburgh Press. 